\title{
Medium range cognitive impairment (MeRCI) hypothesis for psychosis formation: evidence from epidemiological studies and recent molecular genetic developments
}

\author{
Ridha Joober, MD, PhD \\ Douglas Mental Health University Institute, Department of Psychiatry, McGill University, Montréal, Que., Canada
}

Reality always stands in a horizon of desired or feared or, at any rate, still undecided future possibilities. Hence, it is always the case that mutually exclusive expectations are aroused, not all of which can be fulfilled. The undecidedness of the future permits such a superfluidity of expectations that reality necessarily lags behind.

\section{Hans-Georg Gadamer Truth and Method}

Psychotic episodes are a collection of heterogeneous mental states in which patients experience delusions and hallucinations. Delusions are classically defined as "false beliefs based on incorrect inferences about external reality that are firmly sustained despite what almost everyone else believes, ${ }^{\prime 1}$ and hallucinations are perceptions without objects. Although psychotic episodes are characterized by several other symptoms (e.g., disordered thinking, disorganized behaviours), delusions and hallucinations are the most salient and puzzling aspects of the psychotic experience.

The terms that are often used to define psychosis are philosophically very loaded. What are beliefs and external reality and how we make sound inferences have all been and still are some of the most difficult questions in the history of philosophy and science. For some, reality is out there; it does not need to be defined. The expression "losing touch with reality," often used to describe psychosis, is grounded in this conception of reality. For others, reality is coextensive to being human and having expectations, desires and fears. Reality in this sense is not a theatre offered to our senses that we watch in the way that we watch a movie; rather, it is a mental construction in which our memories, emotions, intentions, successes, failures, and conceptions of ourselves and of others are blended and experienced. Thus, the psychotic experience is not a loss of contact with reality, it is just a different form of phenomenal reality.
Although this debate is centuries old and may seem timeworn, we encounter these issues every day in clinical practice, and we engage ourselves in understanding the delusional (and nondelusional) reality of our patients, negotiating with them the need for reflecting on it, and ultimately helping them to remodel it so that it induces less suffering and dysfunction. It is always impressive and sobering to witness how the psychotic experience can differ from patient to patient and how it is difficult to find unifying traits for all these experiences. Delusions can be very difficult to separate, even by experts, from common religious or other cultural beliefs, overvalued ideas, fertile and highly productive imaginations and selfdeception. Alternatively, delusions can be so "out of this world" that their nature is obvious to a nonprofessional observer. They can torment patients and cause them to feel pain, guilt or persecution. They can also be pleasant or even soothing. Delusions may be emotionally charged and drive the person's behaviours or they can be associated with emotional indifference and lack of action. Some delusional patients may be focused on a single persistent theme that takes a major place in their lives (e.g., jealousy, invention, grandiosity), whereas others may go between multiple themes that infiltrate every aspect of their lives. These are only a few aspects of the rich phenomenology of delusions.

Several accounts of the neuropsychological mechanisms of delusions have been proposed. In 1 account, delusions result from failure(s) of some basic neurocognitive or perceptual competencies. ${ }^{2}$ For example, Capgras syndrome, characterized by the belief that familiar persons are replaced by identical-looking imposters, may be conceived primarily as a result of prosopagnosia, a neurocognitive deficit of face recognition due to neural abnormalities in the inferior temporal cortex and its connections with the amygdala. ${ }^{3}$

Correspondence to: R. Joober, Douglas Hospital Research Centre, Frank B. Common Pavilion, 6875 LaSalle Blvd., Verdun QC H4H 1R3; ridha.joober@douglas.mcgill.ca

J Psychiatry Neurosci 2014;39(4):219-22.

DOI: $10.1503 / j p n .140143$ 
Another account of delusion formation relying on neural abnormalities is derived from the prediction error theory. According to this theory, individuals construct expectations about the world and how it should work (i.e., reality), and these expectations are constantly monitored for departure from established regularities. Any unexpected event generates a prediction error signal (encoded at least in part by dopamine burst in the mesolimbic system) that drives attention toward the unexpected happening and allows new interpretations and updated beliefs. In this framework, faulty prediction error signalling due to deregulated dopamine neurotransmission, as documented in schizophrenia, results in major mobilization of attentional recourses and significant attributions to events/ideas or internal representations that will morph into delusions. ${ }^{4}$

A third account of the development of delusions stipulates the existence of cognitive abnormalities, such as reasoning biases $^{5,6}$ (e.g., jumping to conclusion, belief inflexibility, extreme responding) or faulty updating of the probability of already formed hypotheses in the presence of new information. ${ }^{7}$

Evidently, these mechanisms may combine to account for the development and maintenance of delusions. For example, it is possible that abnormal experiences due to cognitive deficits in specific instrumental domains (e.g., face recognition, perceptions of the external world and of the self) may generate abnormal perceptions/experiences, which in the presence of cognitive biases will lead to slanted interpretations that depart substantially from the expected regularities, thus generating strong expectation error signals that may lead to delusion beliefs (for a more detailed discussion see Bortolotti ${ }^{8}$ ).

Hallucinations may be as protean in nature as delusions. They may affect various perceptive modalities, be experienced as "true" perceptions or as unusual and ineffable new perceptual experiences. From a cognitive point of view, parallel theories of faulty concepts (delusions) and faulty percepts (hallucinations) have been put forward. ${ }^{4}$ The rest of this editorial will focus, without significant loss of generalizability, on delusions as a major component of psychotic experience.

This brief discussion of the phenomenology of delusions and the main cognitive theories proposed to explain their mechanisms is not meant to be exhaustive, but rather to highlight that they are, as is likely the case with all psychotic symptoms, very complex psychological constructs rooted in mechanisms that are still poorly understood. Nonetheless, it appears from the above descriptions that the basic cognitive mechanisms supporting the formation of delusions, such as "expectations updating," "jumping to conclusions," "Bayesian updating of priors," "statistical reasoning" and possibly other advanced cognitive schemata, are the same mechanisms involved in normal thinking, although they seem to operate at a suboptimal level, resulting in faulty inferences. From this, one can expect that delusional thinking is more likely to appear under conditions of mild/moderate cognitive deficits that impair inferential thinking so that it results in faulty inferences, but less likely to appear under profound cognitive deficits that obliterate profoundly inferential thinking. Of course, it is also expected that superior cognitive abilities may prevent inferential thinking errors and protect against delusional thinking. This is what we call the "medium range cognitive impairment (MeRCI) hypothesis of psychosis formation."
The rest of this editorial presents some evidence supporting this hypothesis, using epidemiological and molecular genetic data. It focuses on examples from 3 main disorders: Alzheimer disease, intellectual deficits and schizophrenia.

A recent review indicates that the prevalence of delusions in patients with Alzheimer disease vary between $15 \%$ and $76 \% .{ }^{9}$ Remarkably, there is some consistency in the literature indicating that psychotic symptoms, both delusions and hallucinations, tend to happen in the first few years after the onset of the disease and tend not to persist more than several months thereafter. In a recent comprehensive review of psychotic symptoms in patients with Alzheimer disease, it was reported that in most cases psychotic symptoms occur in the first 2 years after onset of illness, plateau in the third year and tend to wane after several months of evolution. ${ }^{10,11}$ There is also consistent evidence of a correlation between cognitive decline and the presence of delusions and hallucinations. ${ }^{10}$ These observations support the idea that psychotic symptoms are more likely to be observed in the early course of dementia, when cognitive abilities are still compatible with inferential thinking, albeit less than optimally, but that they tend to disappear when the cognitive decline deepens to the point that inferential thinking becomes unsustainable.

In the last few years, a number of epidemiological studies sought to determine the prevalence of psychotic symptoms among individuals with intellectual deficits. In the Lundby cohort, the cumulative incidence of psychotic disorders was $8 \%$, which is significantly higher than in the general population. ${ }^{12}$ In this study, psychiatric disorders, including schizophrenia, were more prevalent in individuals with mild than in those with moderate intellectual disability. In another study specifically designed to compare the rate of psychopathology in 2 community groups with varying degrees of intellectual disability, it was reported that psychotic disorders were significantly overrepresented in patients with mild/moderate than in those with severe/profound intellectual deficits. ${ }^{13}$ In a third study comparing the prevalence of psychiatric manifestations in individuals with intellectual deficiencies stratified according to the degree of intellectual challenge, patients with moderate deficits were significantly more likely to present psychotic symptoms, both hallucinations and delusions. ${ }^{14}$ Thus, the MeRCI hypothesis of psychosis formation finds some support.

Schizophrenia is typically defined by the presence of psychotic symptoms. It is now well established that schizophrenia is associated with mild cognitive deficits in different domains and that these deficits often predate the onset of the disorder and may deepen before the onset of psychotic symptoms. In the British cohort, Jones and colleagues ${ }^{15}$ have shown that patients in whom schizophrenia developed had lower educational test scores at ages 8, 11 and 15 years and that there was a correlation between the risk for schizophrenia and test scores. In a historical cohort, Zammit and colleagues ${ }^{16}$ found that high premorbid IQ scores were protective against psychotic disorders. Finally, in the Dunedin cohort, a prospective follow-up study, ${ }_{1}^{17}$ it was reported that individuals in whom schizophrenia developed had a significantly lower IQ than healthy controls (93.63 v. 101.05) before 
the age of 13 years, with a further decline of 6 IQ points between age 13 and adulthood. Thus, it appears that there is a clear consistency across disorders, indicating that psychotic symptoms are more likely to happen within MeRCI.

Interestingly, most of the genetic findings in the field of intellectual deficit, early neurodevelopmental disorders and schizophrenia are compatible with the MeRCI hypothesis of psychosis formation. First, rare and highly penetrant genetic mutations (both point mutations and cytogenetic rearrangements) that have been frequently implicated in individuals with intellectual deficits have very rarely been identified in those with schizophrenia. As these mutations are often responsible for severe mental deficits, it is likely that they impair cognitive functioning to the point that inferential thinking is severely compromised, rendering delusional beliefs impossible to form. In contrast to these severe genetic mutations, in the last few years, a large number of genetic copy number variations (CNVs) have been associated with several neurodevelopmental disorders, most frequently intellectual deficits, but also autism, epilepsy and schizophrenia. In what is probably the largest investigation of these CNVs, Kirov and colleagues ${ }^{18}$ determined the penetrance and the selective pressure imparted by 70 "developmental" CNVs on developmental delay/intellectual deficiency (DD), autism-spectrum disorders (ASD) and various congenital malformations (CM) on the one hand and schizophrenia on the other hand. This analysis was based on 13465 patients with schizophrenia, 32587 patients with $\mathrm{DD} / \mathrm{ASD} / \mathrm{CM}$ and 17873 healthy controls and revealed a very strong correlation between selective pressure (a measure of severity) imparted by these mutations and penetrance (probability of having the phenotype given that the patient is a carrier of the mutation) for DD/ASD/CM $(0.83 ; p<0.001)$. Most interestingly, severe $\mathrm{CNVs}$ that are highly penetrant for DD/ASD/CM (e.g., Angelman/Prader-Willi, Williams-Beuren syndrome, 1q36 deletion syndrome) were not observed in patients with schizophrenia, which strongly suggests that psychotic disorders do not develop in the context of severe/highly penetrant mutations that cause severe intellectual deficits. Seventeen CNVs were reported to be associated with a significantly increased risk for schizophrenia. In carriers of these CNVs, the risk for DD/ASD/CM ranged from 13\% to $100 \%$, whereas their risk for schizophrenia was much lower (average of 6 times lower), ranging from $2 \%$ to $18 \%$. This decreased risk for schizophrenia compared with DD/ASD/CM was true for each CNV. This suggests that the intellectual deficits imparted by these mutations represent the background for the development of psychotic disorders. Most interestingly, calculating the correlation between the penetrance for $\mathrm{DD} / \mathrm{ASD} / \mathrm{CM}$ and the penetrance for schizophrenia in the $17 \mathrm{CNVs}$ associated with schizophrenia revealed that this correlation is significant $(r=$ $0.66 ; p=0.007$ ), reinforcing the idea that the risk for psychosis in carriers of these CNVs is a function of their impact on intellectual development.

From a genetic point of view, the obvious question then is why some patients with these intermediate penetrance mutations transition to psychosis but most do not. It is of course possible that carrying additional CNVs rather than only $1 \mathrm{CNV}$ may increase the risk for psychosis. However, if the
MeRCI hypothesis presented here is correct, individuals with a higher load of CNVs would have greater developmental delays/intellectual deficits, thus they might be less likely to present psychotic symptoms. There is in fact some evidence to suggest that this is true. In a very large study $(n=32587)$ by Girirajan and colleagues ${ }^{19}$ specifically designed to test the effect of multiple CNVs on the phenotypic expression of developmental disorders, it was reported that median IQ correlates with the number of genes disrupted by the CNVs (which correlates with the number of CNVs). It was also reported that in patients having multiple hits, the severity of the developmental disorder is much greater, but not a single case of schizophrenia or psychotic disorder has been reported. Interestingly, Kirov and colleagues ${ }^{18}$ also tested the multiple hit hypothesis and reported no additional CNVs in patients with schizophrenia compared with those who did not have the disorder. Thus the question remains why some $\mathrm{CNV}$ carriers transition to psychosis and others do not. However, if the MeRCI hypothesis for psychosis formation is correct, the answer to this question should not involve higher genetic load affecting the cognitive functioning.

Finally, in a genome-wide association study (GWAS) investigating genetic variations with high frequency and very low penetrance (single nucleotide polymorphisms [SNPs]), it has been shown that patients with schizophrenia have lower cognitive polygenic scores than controls and that the polygenic scores for schizophrenia were associated with lower general cognitive ability ${ }^{20}$ In addition, in this same study, many of the SNPs that were robustly associated with schizophrenia through GWASs were linked with mild cognitive deficits, a result that echoes many other studies that reported an association between GWAS schizophrenia SNPs and lower cognitive abilities..$^{21,22}$

Some implications for the understanding of the genetics of psychosis may be gleaned from the MeRCI hypothesis for psychosis formation and the genetic literature discussed here. Indeed, it might be argued that CNVs may be the most informative genetic variations with regard to the mechanisms of psychotic disorders. This is because the penetrance of CNVs with regard to psychosis is several times greater than the penetrance of SNPs implicated in schizophrenia. In the absence of causative rare and highly penetrant mutations, ${ }^{23}$ it may be assumed that the mechanisms imparted by CNVs toward psychosis will be the most informative from a genetic point of view. Thus any understanding of the mechanisms by which CNVs lead to psychosis will be of the highest value. It appears that the involvement of CNVs in psychotic symptoms is contingent on their effects on cognition in a rather nonspecific fashion. Indeed, the high and significant correlation between the penetrance of CNVs for schizophrenia and for $\mathrm{DD} / \mathrm{ASD} / \mathrm{CM}$ in conjunction with the much higher penetrance of these mutations to $\mathrm{DD} / \mathrm{ASD} / \mathrm{CM}$ compared with their penetrance for schizophrenia strongly favour a nonspecific effect (mainly through a reduction of the cognitive abilities to the medium range) of these mutations. This brings us to the interesting question of whether the genetics of schizophrenia could be explained to a large extent, if not entirely, by the genetics of the MeRCI. 
The major clinical implication of the MeRCI hypothesis for psychosis formation is that patients with psychotic disorders have cognitive strengths. The presence of delusions in patients with psychosis may be considered as a testimony to a preserved, albeit error-prone, inferential thinking. This of course gives a strong justification for the utility of cognitive remediation. In fact, there is now a large literature indicating that cognitive therapy is effective in many forms of psychotic states. ${ }^{24}$ Another clinical implication of this hypothesis is the possibility of targeting youth within the MeRCI for indicated prevention of psychotic disorders. Up until now, studies investigating groups at high risk for psychotic disorders did not include much consideration for cognitive abilities. It will be interesting to monitor conversion rates in youth at high risk for psychotic disorders as a function of their cognitive abilities or the change of their cognitive abilities over time. Alternatively, it will be interesting to develop specific interventions targeting faulty inferential thinking and to test their efficacy in preventing conversion to psychotic states in patients with high-risk states who show dysfunctional inferential thinking.

This editorial has covered a number of issues. First, there is no doubt that delusions and other psychotic symptoms are very complex and difficult to grasp within a simple framework. Second, using the neuropsychological account of delusions, I propose that delusions, and possibly other aspects of the psychotic symptoms, are more likely to emerge under a MeRCI, although these are neither necessary nor sufficient to the development of psychosis. Third, given that the association between this MeRCI and psychotic symptoms is observed under a large array of pathological conditions (neurodegenerative and neurodevelopmental) and genetic mutations, it is very likely that the basic mechanisms that predispose to psychosis are extremely heterogeneous, but they tend to funnel into cognitive deficits that are compatible with preserved, although quite error-prone, inferential thinking. However, it remains puzzling that psychotic disorders can afflict the brightest among us (John Nash is a famous example). Even in the absence of cognitive bias or intellectual deficits, human beings may become delusional. As Gadamer posited, reality always lags behind the undecidedness of the future and the superfluidity of our expectations. Desires, aspirations and fears of our undecided future may transform this lagging reality into delusions, particularly when our biological/ genetic and personal histories aid this transformation.

\section{References}

1. American Psychiatric Association. Diagnostic and statistical manual of mental disorders. 5 ed. Washington (DC): The Association; 2013.

2. Maher BA. Delusional thinking and perceptual disorder. I Individ Psychol 1974;30:98-113.

3. Ramachandran VS. Consciousness and body image: lessons from phantom limbs, Capgras syndrome and pain asymbolia. Philos Trans R Soc Lond B Biol Sci 1998;353:1851-9.

4. Adams RA, Stephan KE, Brown HR, et al. The computational anatomy of psychosis. Front Psychiatry 2013;4:47.
5. Garety PA, Freeman D, Jolley S, et al. Reasoning, emotions, and delusional conviction in psychosis. J Abnorm Psychol 2005;114:373-84.

6. Garety PA, Freeman D. The past and future of delusions research: from the inexplicable to the treatable. Br J Psychiatry 2013;203:327-33.

7. Langdon R, Ward PB, Coltheart M. Reasoning anomalies associated with delusions in schizophrenia. Schizophr Bull 2010;36:321-30.

8. Bortolotti L. Delusion. The Stanford encyclopedia of philosophy [serial online]. 2013. Available: http://plato.stanford.edu/ (accessed 2014 June 3).

9. Cipriani G, Danti S, Vedovello M, et al. Understanding delusion in dementia: a review. Geriatr Gerontol Int 2014;14:32-9.

10. Paulsen JS, Salmon DP, Thal LJ, et al. Incidence of and risk factors for hallucinations and delusions in patients with probable AD. Neurology 2000;54:1965-71.

11. Ropacki SA, Jeste DV. Epidemiology of and risk factors for psychosis of Alzheimer's disease: a review of 55 studies published from 1990 to 2003. Am J Psychiatry 2005;162:2022-30.

12. Nettelbladt P, Goth M, Bogren M, et al. Risk of mental disorders in subjects with intellectual disability in the Lundby cohort 1947-97. Nord J Psychiatry 2009;63:316-21.

13. Myrbakk E, von TS. Screening individuals with intellectual disability for psychiatric disorders: comparison of four measures. Am J Ment Retard 2008;113:54-70.

14. Holden B, Gitlesen JP. The association between severity of intellectual disability and psychiatric symptomatology. J Intellect Disabil Res 2004;48:556-62.

15. Jones P, Rodgers B, Murray R, et al. Child development risk factors for adult schizophrenia in the British 1946 birth cohort. Lancet 1994;344:1398-402.

16. Zammit S, Allebeck P, David AS, et al. A longitudinal study of premorbid IQ Score and risk of developing schizophrenia, bipolar disorder, severe depression, and other nonaffective psychoses. Arch Gen Psychiatry 2004;61:354-60.

17. Meier MH, Caspi A, Reichenberg A, et al. Neuropsychological decline in schizophrenia from the premorbid to the postonset period: evidence from a population-representative longitudinal study. Am J Psychiatry 2014;171:91-101.

18. Kirov G, Rees E, Walters JT, et al. The penetrance of copy number variations for schizophrenia and developmental delay. Biol Psychiatry 2014;75:378-85.

19. Girirajan S, Rosenfeld JA, Coe BP, et al. Phenotypic heterogeneity of genomic disorders and rare copy-number variants. N Engl J Med 2012;367:1321-31.

20. Lencz T, Knowles E, Davies G, et al. Molecular genetic evidence for overlap between general cognitive ability and risk for schizophrenia: a report from the Cognitive Genomics consorTium (COGENT). Mol Psychiatry 2014;19:168-74.

21. Albanna A, Choudhry Z, Harvey PO, et al. TCF4 gene polymorphism and cognitive performance in patients with first episode psychosis. Schizophr Res 2014;152:124-9.

22. Cassidy C, Buchy L, Bodnar M, et al. Association of a risk allele of ANK3 with cognitive performance and cortical thickness in patients with first-episode psychosis. J Psychiatry Neurosci 2014;39:31-9.

23. Purcell SM, Moran JL, Fromer M, et al. A polygenic burden of rare disruptive mutations in schizophrenia. Nature 2014;506:185-90.

24. Barlati S, Deste G, De PL, et al. Cognitive remediation in schizophrenia: current status and future perspectives. Schizophr Res Treatment 2013;2013:156084 